\title{
THE MACROECONOMIC ASPECTS OF SPORT IN EUROPE: A CROSS-COUNTRY COMPARISON
}

\section{INTRODUCTION}

Sport is an immanent part of modern lifestyle in contemporary societies. The sports sector of the economy is becoming increasingly important in middle- and high-income countries. Besides the social value of physical activities, sport has an economic value and contributes to the national economy: to economic growth and the labour market, with value added and employment effects exceeding average growth rates. ${ }^{1}$ However, the macroeconomic effects sports are not fully explained and measured. Due to the specific definition of sport $^{2}-$ it is not considered as a separate industry or branch. The complexity of sport-related activities and markets makes the assessment of sport's contribution to economic growth and the national labour market difficult. Statistical measurement of the sports sector is scarce and quite complicated. Data collections on sport cover participation issues or regional or national statistics about sports objects. European Union (EU) level Sport Satellite Accounts (SSAs) provides reliable data on the economic impact of sport on economic growth in the EU Member States (MSs). However, the SSAs are produced every four years and there are some MSs that are slow to include SSAs in the national statistics. Eurostat provides more frequent (but also more superficial) examinations of the sports sector's contribution to economic growth.

In this examination we aim to investigate the macroeconomic importance of sport and sport-related activities in the European Union Member States. We would like to characterize the effect of the sports sector on some macrolevel indicators. It would reveal, to some degree, the power of the sports sector to affect aggregate results. We refer to them as to the sports economics, which makes clear our economic (not social nor cultural) approach to sport analysis. We discuss data provided by Eurostat and, in addition, the results of the Sport Satellite Accounts-based examinations (or their approximation). We explain our choice of data sources for analysis in the body of the manuscript. Moreover, we aim to juxtapose the national results of MSs. We expect to find rising importance of the sports sector over time, as well as a greater contribution from sports in developed economies.

1 Commission of the European Communities, White Paper on Sport, Brussels, COM(2007)391 final: 10 .

${ }^{2}$ There are several definition of sport. We present them in the second section of the paper. 
The research period of our examination is 2011-2018. Eurostat provides a special section of sport-related data which mainly dates back to 2011. Additionally, we present the economic impact of sport based on 2012 Sport Satellite Accounts. We do not have more recent results of SSAs at our disposal. As they present contribution of the sports sector to the gross domestic product (GDP) or value added in the most comprehensive manner, we decided to present 2012 data as well.

We were motivated to conduct this examination by the scarcity of research publications that search for the impact of the sports sector on national economies. The reports of SSAs contain statistical interpretation, rather than economic. Moreover, our contribution is related to studying coverage of the EU Member States and the most recent statistics (if they are available). We find the scope of the research interesting, as sport is becoming an increasingly important part of human life and wellbeing. Its examination is worthy of attention.

The article is organized as follows: the second section presents sport economics: definitions of sport and possible impact of the sports sector on macro-level economic performance. The third section briefly characterizes the measurement of the contribution of the sports sector to economic growth. The fourth section compares the results of statistical examination: SSAs-based and the Eurostat data in a cross-country context. Finally, we summarize our findings.

\section{THE SPORTS ECONOMICS}

The increasing role of sport in the lives of societies can be observed. More and more attention is paid to the role of sports activity for individuals, families, and local and national communities. Lack of physical activity (or its low level), inadequate nutrition and stress lead to many diseases, including cancer. Movement is a tremendous means of promoting health. Without active leisure time it is difficult to maintain natural immunity to diseases or psychophysical fitness into old age. ${ }^{3}$ Physical activity is considered to be the best nonpharmacological method for the prevention of most diseases that are currently the main cause of death - the so-called lifestyle diseases. ${ }^{4}$ Nowadays, the concept of work-life balance is also gaining popularity. Sport is becoming a way of pursuing non-professional activities. However, analysing the importance of sports for modern societies does not fall within the scope of this examination. We mention it just to underline that it is gaining increasing significance in various aspects. It does not matter whether we discuss professional sport, large-scale investments in sport facilities or local fitness markets: they all contribute to the national economy. The extension and globalization of the sports sector could be attributed to two factors: annually paid holidays for workers were introduced to national legislation, then television broadcasting followed

\footnotetext{
${ }^{3}$ Cyganiuk (2011): 85.

4 Kozłowska et al. (2015): 356.
} 
by an emergence of new information and communication technology popularized sport and its impact on GDP become more and more remarkable. ${ }^{5}$ However, the sports sector as a whole is not a separate, statistically measured sector. It is part of various other industries and economic sectors. A qualitative analysis of the multidimensional impact of the sports sector on the economy was presented by Gratton. ${ }^{6}$

Moreover, even the definition of sport is rather complicated. It was unified on the EU level in 2007 (Table 1). The so-called Vilnius definitions of sport (adopted in Vilnius during one of the meetings of the Working Group on Economy and Sport) are statistical, narrow and broad definitions. Each subsequent definition is an extension of the previous one. The broad definition shows sport in its fullest and most comprehensive dimension, including both competitive and recreational sports ${ }^{7}$. In national law, sport is not always defined separately, and EU Member States usually refer to the Vilnius definition.

\section{Table 1}

Definition of sports

\begin{tabular}{|l|l|}
\hline $\begin{array}{c}\text { Coverage } \\
\text { of the definition }\end{array}$ & \multicolumn{1}{c|}{ Sport interpretation } \\
\hline Statistical & $\begin{array}{l}\text { Refers to the NACE (Statistical Classification of Economic Activities in } \\
\text { the European Community) 92.6 classification: activities directly related } \\
\text { to sports } \\
\text { It includes the activities of sports infrastructure: stadiums and other } \\
\text { sports facilities as well as services related to the organization and pro- } \\
\text { motion of sporting events }\end{array}$ \\
\hline Narrow & $\begin{array}{l}\text { It includes the activities of all sectors in the economy that produce goods } \\
\text { and provide services necessary for practicing sport }\end{array}$ \\
\hline Broad & $\begin{array}{l}\text { It includes all essential industries and activities directly or indirectly } \\
\text { related to sport for which sport is a significant contribution to their func- } \\
\text { tioning (e.g. television broadcasts, the production of computer games) }\end{array}$ \\
\hline $\begin{array}{l}\text { White Paper } \\
\text { on Sport }\end{array}$ & $\begin{array}{l}\text { All forms of physical activity which, through casual or organized partici- } \\
\text { pation, aim at expressing or improving physical fitness and mental well- } \\
\text { being, forming social relationships or obtaining results in competition at } \\
\text { all levels }\end{array}$ \\
\hline $\begin{array}{l}\text { Economic point } \\
\text { of view }\end{array}$ & $\begin{array}{l}\text { Sport is an activity which has repercussions in many different areas of } \\
\text { the economy }\end{array}$ \\
\hline
\end{tabular}

Source: the authors' own study based on: European Commission, Sport Satellite Accounts. A European Project: New Results, 2013: 2; Study commissioned by the European Commission, DirectorateGeneral Education and Culture, Study on the Contribution of Sport to Economic Growth and Employment in the EU, 2012: 13; Commission of the European Communities, White Paper on Sport, Brussels, COM(2007)391 final: 2.

${ }^{5}$ Andreff (2008): 13-14.

${ }^{6}$ Gratton (1998): 101-117.

${ }^{7}$ Competitive sport focuses on achieving good results and medals. With recreational sport, the point is relaxation, contact with others, or maintaining physical fitness that affects health. 
Our examination covers sport and its contribution to economic performance. This is why we refer rather to the broad understanding of sport. It covers sports both as an industry (production sector) and service. At the same time, it is more precise than a simple economic point of view on sports (last definition), which makes it possible to present economic statistics related to sport activities. As we have already mentioned, the sports sector is not an isolated industry. This has consequences for the measurement of the sports sector's contribution to the economy. The Study on the Contribution of Sport to Economic Growth and Employment in the $E U$ provides a list of some sport-related activities and products with an economic impact. This report distinguishes three groups of such activities and products: ${ }^{8}$ (1) Goods and services conditional on doing sports, (2) Doing sports, (3) Goods and services necessary to do sport. The first group is the most diversified, as it covers, for example: dietary supplements, sport tourism facilities, sport bets, health services, and veterinarian services. The second group mimics the statistical definition of sport. Here the issue is sport-related objects and professional sports. The last group covers a variety of categories, such as fitness centres, dance schools, sports clothing, or sport-related education. A macroeconomic point of view for such a variety of goods, services and industries is that their production and sale create value added and jobs where they are provided.

To include a value in GDP accounting, it does not matter where the added value is created. Demand for sports-related goods and services is generated by all groups of economic agents, primary by households. Regardless of the domination of households as a demand-creating group, demand for sport activities and products comes from companies: public sector and abroad (export).

Once we have analysed the importance of sports for the macro results of the national economy, we should point out the areas of sports contribution. The most obvious way is to consider sport and its contribution to the GDP (or value added). Secondly, employment in the sports sector could be considered. More detailed approaches are offered by the analysis of private and public spending or the examination of international trade.

We expect to see the positive and rising contribution of the sports sector to macro-level results. This is due to the increasing importance of sport in the everyday life of citizens of medium- and high-income level countries. However, there are some situations when the effects do not meet expectations. Hosting the Olympics or one of the big international football tournaments is a case in point. Positive economic benefits for the short and long term are expected by supporters of regional or national involvement in mega sport events. The event is expected to stimulate economic activity, increase export revenues ${ }^{9}$ (for example from tourism), create positive publicity for the country, and in turn lead to economic growth and increased levels of domestic and foreign invest-

8 SportsEconAustria (SpEA), Study on the Contribution of Sport to Economic Growth and Employment in the EU. 2012. <https://publications.europa.eu/en/publication-detail/-/publication /865ef44c-5ca1-11e8-ab41-01aa75ed71a1/language-en/format-PDF/source-71256399> [accessed 15 June 2019]: 3.

9 Rose and Spiegel (2011): 667. 
ment. ${ }^{10}$ However, as a broad strand of the literature presents, the economic benefits of mega sport events of various natures are usually overestimated. ${ }^{11}$

In this examination we present macro statistics generally describing the sports sector without any explicit reference to the effects of mega event.

\section{AN EVALUATION OF SPORT'S MACROECONOMIC RELEVANCE}

Sport is a significant sector of any economy, even though it is seldom included in statistics, which makes it difficult to prepare analyses connected with this topic. This essentially applies to its economic and financial aspect, ${ }^{12}$ as there are no such problems from the point of view of registering results at the professional sport level.

The analysis of the relationship between sport and the economy concerns both the micro and macroeconomic levels. The sports market consists of two areas where active (the so-called sports market) and passive (the so-called spectators market) sports consumption continues. In the case of the active side, we discuss suppliers (public sector, private sector, volunteering), products (for example training offers) and buyers, but also markets regarding license, media, advertising and sponsorship rights. When discussing the passive side, one should consider: buyers (consumers), products (for example sports performances) and suppliers (for example organizers of sports competitions). ${ }^{13}$

To address sport and the economy, the European Commission created the Working Group on Sport and Economics in 2006. Its purpose was to elaborate the European method of measuring the impact of sport on the economy and creating a European Sport Satellite Account, which will be discussed later in the article. Sport Satellite Accounts have so far been prepared for nine EU MSs (Austria, Belgium, Cyprus, Germany, Lithuania, the Netherlands, Portugal, Poland, and the United Kingdom). Luxembourg and Croatia are preparing to launch SSAs. The unavailability of coherent data for the EU also highlights the difficulties related to the examination of the sports sector.

Cyprus, the Czech Republic, the Netherlands and Germany prepare SSAs irregularly. There are also those Member States that have not yet produced any SSA feeds, which unfortunately hinders research into the detailed determination of the economic importance of sport for the economies of all EU countries. ${ }^{14}$

National accounts are the most important reference statistics for economic policy on the macro level and are normally conducted by the nation-

\footnotetext{
10 Jacobsen et al. (2013): 3778-3779.

${ }^{11}$ See Porter and Fletcher (2008); Allmers, Maennig (2009); Matheson (2009); Jacobsen et al. (2013).

12 There are publications that draw attention to the relationship between the world of sport and the world of finance, e.g. Siwiński, Tauber, Mucha-Szajek (2009).

${ }^{13}$ Grabowski (2014):190.

${ }^{14}$ Grabowski (2017): 2, 8.
} 
al statistical office. They could be extended by a satellite account. 'A Sport Satellite Account - being the core of an Input-Output Table: Sport - filters the national accounts for sport-relevant activities to extract all sport-related figures while maintaining the structure of the national accounts. The instrument of SSAs permits all sport-related economic activities to show up explicitly, rather than keeping them concealed, in deeply disaggregated (low level) classifications of the national accounts. ${ }^{15}$ The production of sport shoes is a clear example here. It is classified as a part of 'textiles, wearing apparel, leather and related products'. The Sport Satellite Accounts provide separate sectors for sport shoes. 'This implies strict non-additivity of satellite accounts which is often ignored in public discussions. As an example, sport-related tourism is a part of the sport satellite account. However, it also shows up in tourism satellite accounts. Adding the effects of both satellite accounts would double count sport-related tourism. Because of that, satellite accounts must always be interpreted as stand-alone models. ${ }^{16}$ Satellite accounts are detailed sectoral views on the parts of an economy which would otherwise remain invisible within their main sectors.

A Sport Satellite Account provides the possibility to analyse the contribution of sport to economic and productivity growth, and the growth rates of the sport-relevant parts of the sectors themselves..$^{17}$ Two reports with modelbased, secondary assessments are provided: the Study on the Contribution of Sport to Economic Growth and Employment in the EU, ${ }^{18}$ and the Study on the Economic Impact of Sport through Sport Satellite Accounts. ${ }^{19}$ They extend SSAs' information and clarify the sport-related contribution to GDP (or gross value added) and employment. These reports cover all EU Member States. For countries that do not prepare an SSA, alternative sources of data are used, accompanied by estimations based on the results obtained for SSA-covered countries with a comparable economic structure. A starting point of the Reports' estimation was setting up a Multiregional Input-Output Table: Sport which is a transformation of national Input-Output Tables.

The authors of both assessments aimed to estimate growth rates. Such a dynamic approach needed SSAs results from two different periods. The EU-wide

${ }^{15}$ Study commissioned by the European Commission, Directorate-General Education and Culture, Study on the Contribution of Sport to Economic Growth and Employment in the EU, 2012: 1 .

${ }^{16}$ European Commission, Study on the Economic Impact of Sport through Sport Satellite Accounts, 2018: 15 .

17 SportsEconAustria (SpEA), Study on the Contribution of Sport to Economic Growth and Employment in the EU, 2012, <https://publications.europa.eu/en/publication-detail/-/publication /865ef44c-5ca1-11e8-ab41-01aa75ed71a1/language-en/format-PDF/source-71256399> [accessed 15 June 2019]: 66.

18 SportsEconAustria (SpEA), Study on the Contribution of Sport to Economic Growth and Employment in the EU, 2012, <https://publications.europa.eu/en/publication-detail/-/publication /865ef44c-5ca1-11e8-ab41-01aa75ed71a1/language-en/format-PDF/source-71256399> [accessed 15 June 2019].

19 SportsEconAustria (SpEA), Study on the Economic Impact of Sport through Sport Satellite Accounts, 2018, <https://ec.europa.eu/assets/eac/sport/library/studies/study-contribution-spors-economic-growth-final-rpt.pdf> [accessed 15 June 2019]. 
SSAs were not available when the reports were prepared. An alternative procedure to capture direct and indirect effects of sport-related sectors was applied: output multipliers based on some relatively small sport-related sectors that have high national multipliers. ${ }^{20}$ The idea of allying multipliers to capture the effect of the sports sector for aggregate output was previously presented in economic studies. ${ }^{21}$ Growth potentials were found in high-multipliers sectors. To estimate sports economy contribution to growth or value added, the authors also identified the key sectors of the economies covered to find drivers of regional growth and the range of products to analyse country's possible dependence on imports.

The results of these SSAs examinations are provided in two reports which we mention above. We use the results presented in these reports to discuss the contribution of sports to the GDP and gross value added. They are based on SSAs; the pure results of SSAs are supported by different sources of data and model values in question.

The analysis of the sports sector with the application of SSAs would be an optimal choice for economists. This is why we characterized SSAs and their possible extensions in the first place. However, due to the scarcity, frequency and partial coverage of the EU MSs by satellite accounts, our analysis is mainly based on more the standard statistical collection provided by the Eurostat.

The Eurostat database, as the source of data that describes the macro impact of sports economy, is quite obvious. Nonetheless, as sport is not an isolated industry or there is no other possibility to separate active and passive sport consumption, special attention was necessary to prepare the sports database. Eurostat uses the Vilnius definitions of sport as a starting point for distinguishing sports-related activities. Sports statistics for the EU Member States are not collected by a single stand-alone survey but come from several data collections (like Labour Force Surveys, Structural Business Statistics, Households Budget Surveys or Harmonised Index of Consumer Prices). Sports statistics concern both the social and economic aspects of sport. They cover information related to sports participation - which is not directly linked to the macro approach of this study or data on sport in the cities. Additionally, the collection of sports data delivers statistics on: ${ }^{22}$

- employment in sport,

- the characteristics and performance of enterprises engaged in the sports sector and the sales of sporting goods,

- international trade in sporting goods,

- private household expenditure on sporting goods and services,

- the price index of sporting goods and services.

20 SportsEconAustria (SpEA), Study on the Contribution of Sport to Economic Growth and Employment in the EU, 2012, < https://publications.europa.eu/en/publication-detail/-/publication /865ef44c-5ca1-11e8-ab41-01aa75ed71a1/language-en/format-PDF/source-71256399> [accessed 15 June 2019]: 68.

${ }^{21}$ Gibson, McIntyre, MacKay \& Riddington (2005): 321-329.

22 Eurostat Sports Collections: <https://ec.europa.eu/eurostat/web/sport/data/database> [accessed 20 June 2019]. 
Still, the data available in the sports section does not make it possible to assess directly the impact of the sports sector on economic growth, which is more of an issue with Sport Satellite Accounts. However, they provide information on important macro issues, such as: employment, consumption expenditures, prices, international exchange. In the next section we compare Eurostat statistics with the SSAs findings.

\section{A CROSS-COUNTRY COMPARISON OF THE ECONOMIC SIGNIFICANCE OF SPORT IN THE EU MEMBER STATES}

We choose four statistics provided by the Eurostat and SSAs-based estimations of the contribution of the sports sector to GDP and the value added to present the importance of sport for national economies of the EU Member States. Employment in sport (Table 2) expressed as a percentage of total employment varies from about $0.3 \%$ to about $1.7 \%$. Generally it increases over time and is higher in the older EU Member States. As tourism is classified as a sports activity, it may be surprising that sport employment is relatively low in some countries recognized as highly tourist-oriented, such as Greece or Italy. This could be explained as follows: a large portion of tourism employees work illegally - being for instance a part of family business - and this is not captured by the official statistics.

Table 2

Employment in sport

\begin{tabular}{|l|c|c|c|c|c|c|c|c|}
\hline MS/TIME & $\mathbf{2 0 1 1}$ & $\mathbf{2 0 1 2}$ & $\mathbf{2 0 1 3}$ & $\mathbf{2 0 1 4}$ & $\mathbf{2 0 1 5}$ & $\mathbf{2 0 1 6}$ & $\mathbf{2 0 1 7}$ & $\mathbf{2 0 1 8}$ \\
\hline EU-28 & 0.70 & 0.73 & 0.70 & 0.72 & 0.74 & 0.76 & 0.76 & 0.77 \\
\hline AUT & 0.62 & 0.59 & 0.63 & 0.67 & 0.87 & 0.66 & 0.62 & 0.56 \\
\hline BEL & 0.53 & 0.51 & 0.47 & 0.45 & 0.44 & 0.53 & 0.50 & 0.49 \\
\hline BGR & 0.35 & 0.30 & 0.31 & 0.32 & 0.35 & 0.39 & 0.42 & 0.41 \\
\hline HRV & 0.25 & 0.29 & 0.30 & 0.39 & 0.30 & 0.38 & 0.39 & 0.59 \\
\hline CYP & 0.82 & 0.56 & 0.53 & 0.58 & 0.53 & 0.76 & 0.77 & 0.61 \\
\hline CZE & 0.53 & 0.45 & 0.51 & 0.58 & 0.54 & 0.61 & 0.63 & 0.64 \\
\hline DNK & 1.00 & 0.97 & 0.92 & 1.04 & 1.09 & 1.17 & 0.98 & 1.02 \\
\hline EST & 0.56 & 0.65 & 0.82 & 1.01 & 0.77 & 0.87 & 0.80 & 0.82 \\
\hline FIN & 0.96 & 0.96 & 1.06 & 1.27 & 1.26 & 1.26 & 1.27 & 1.26 \\
\hline FRA & 1.09 & 1.08 & 0.83 & 0.64 & 0.67 & 0.69 & 0.64 & 0.69 \\
\hline DEU & 0.45 & 0.52 & 0.55 & 0.55 & 0.56 & 0.59 & 0.61 & 0.61 \\
\hline GRC & 0.31 & 0.22 & 0.28 & 0.40 & 0.43 & 0.47 & 0.53 & 0.56 \\
\hline
\end{tabular}




\begin{tabular}{|l|l|l|l|l|l|l|l|l|}
\hline HUN & 0.29 & 0.29 & 0.31 & 0.35 & 0.44 & 0.39 & 0.36 & 0.42 \\
\hline IRL & 1.05 & 0.98 & 1.03 & 1.02 & 0.95 & 1.09 & 1.08 & 1.08 \\
\hline ITA & 0.46 & 0.44 & 0.47 & 0.54 & 0.52 & 0.52 & 0.57 & 0.50 \\
\hline LVA & 0.55 & 0.69 & 0.54 & 0.65 & 0.62 & 0.67 & 0.67 & 0.93 \\
\hline LTU & 0.42 & 0.41 & 0.41 & 0.45 & 0.51 & 0.57 & 0.54 & 0.57 \\
\hline LUX & 0.51 & 0.58 & 0.65 & 0.49 & 0.75 & 1.07 & 0.82 & 0.75 \\
\hline MLT & 0.38 & 0.52 & 0.67 & 0.66 & 0.54 & 0.35 & 0.68 & 0.77 \\
\hline NLD & 0.87 & 0.86 & 0.86 & 0.89 & 0.97 & 1.00 & 0.92 & 0.96 \\
\hline POL & 0.29 & 0.32 & 0.34 & 0.37 & 0.36 & 0.40 & 0.42 & 0.46 \\
\hline PRT & 0.50 & 0.48 & 0.64 & 0.71 & 0.85 & 0.85 & 0.84 & 0.77 \\
\hline ROU & 0.13 & 0.23 & 0.16 & 0.12 & 0.14 & 0.15 & 0.17 & 0.12 \\
\hline SVK & 0.29 & 0.37 & 0.51 & 0.53 & 0.46 & 0.47 & 0.46 & 0.38 \\
\hline SVN & 0.56 & 0.44 & 0.46 & 0.45 & 0.61 & 0.58 & 0.60 & 0.56 \\
\hline ESP & 0.80 & 0.91 & 0.96 & 1.08 & 1.03 & 1.05 & 1.07 & 1.09 \\
\hline SWE & 1.26 & 1.36 & 1.51 & 1.54 & 1.60 & 1.53 & 1.60 & 1.65 \\
\hline GRB & 1.29 & 1.35 & 1.23 & 1.30 & 1.35 & 1.37 & 1.37 & 1.39 \\
\hline
\end{tabular}

Note: Percentage of total employment. We use three-letter ISO country codes to denote MSs in this and subsequent tables. MS are presented in alphabetical order.

Source: Eurostat Sports Collections, <https://ec.europa.eu/eurostat/web/sport/data/database> [accessed 20 June 2019].

Secondly, we would like to discuss the sports industry: manufacture of sports goods (Table 3). The Statistical Classification of Economic Activities in the European Community (NACE) and national accounts system make it possible to directly derive data only about this fraction of the sports sector. According to the statistics on value added, sports goods production generates the highest value added in Austria, France, Germany, Italy, and the UK. Again, these are developed economies, where some well-known sports brands are located. Adidas factories, which are spread throughout Europe, could be a good example here: more than twenty of them are located in Germany, more than ten in Italy and Spain. Austria hosts some well-recognized producers of ski equipment. Germany has a considerable sports nutrition sector - much more important than any remaining EU MS. Even if the number of sports goods producers fluctuates over time in some economies, their number, once we consider the European level, increased substantially since 2011. The highest level of value added by sports goods producers is not necessarily accompanied by the highest number of enterprises classified as manufacturers of sports goods.

International trade statistics (Table 4) mimic to some extent the statistics on the value added by sport goods: France, Germany and Italy are again recording the most significant turnovers of imports or exports with international 


\begin{tabular}{|c|c|c|c|c|c|c|c|c|c|c|c|c|c|c|c|c|c|c|c|c|c|}
\hline స్ & \multirow{7}{*}{ 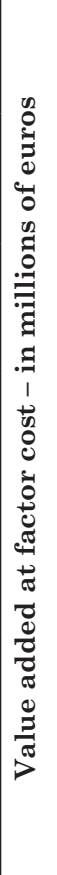 } &.. & $\begin{array}{l}\stackrel{\sigma}{ } \\
\dot{0} \\
\stackrel{1}{ }\end{array}$ &.. &.. & $\stackrel{\ddot{1}}{\ddot{\infty}}$ & 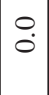 & .. &.$\cdot$ & 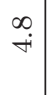 &.. & $\begin{array}{l}\sim \\
\infty \\
\infty \\
\infty \\
\infty\end{array}$ & $\begin{array}{l}\sigma \\
0 \\
0 \\
\infty \\
\infty\end{array}$ & $\stackrel{\wp}{\sim}$ & $\begin{array}{l}H \\
\dot{\omega}\end{array}$ &.. &.. & $\begin{array}{c}0 \\
\stackrel{\sim}{v}\end{array}$ & $\vec{i}$ &.. & .. \\
\hline $\begin{array}{l}0 \\
\stackrel{0}{0} \\
\text { Oे }\end{array}$ & & $\begin{array}{l}\infty \\
\infty \\
0 \\
\sim\end{array}$ & $\begin{array}{l}\overrightarrow{+} \\
\vec{\infty} \\
\vec{N}\end{array}$ & $\begin{array}{l}\infty \\
\dot{0} \\
\dot{0}\end{array}$ & $\stackrel{\sim}{\vec{N}}$ & $\stackrel{+}{\ddot{\infty}}$ & $\ddot{0}$ & $\mid \begin{array}{r}\vec{\sigma} \\
\dot{\sigma}\end{array}$ & $\cdot \cdot$ & $\stackrel{\infty}{+}$ & $\begin{array}{l}\infty \\
\dot{\forall} \\
\dot{\forall}\end{array}$ & $\begin{array}{l}\infty \\
\ddot{0} \\
\stackrel{0}{0} \\
\infty\end{array}$ & $\begin{array}{c}10 \\
\dot{+} \\
\stackrel{\infty}{\infty} \\
\stackrel{\infty}{0}\end{array}$ & $\stackrel{\circ}{\sim}$ & $\begin{array}{l}\overrightarrow{1} \\
\infty \\
\infty \\
\infty\end{array}$ & $\cdot$ & 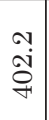 & $\begin{array}{l}\vec{\Delta} \\
\stackrel{i}{*}\end{array}$ & $\cdots$ & $\ddot{0}$ & 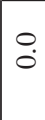 \\
\hline$\stackrel{10}{\stackrel{2}{\circ}}$ & & $\begin{array}{l}0 \\
18 \\
0 \\
-1\end{array}$ & $\begin{array}{l}\infty \\
\stackrel{\infty}{0} \\
\stackrel{\sim}{0}\end{array}$ & $\begin{array}{l}0 \\
\dot{0}\end{array}$ & $\begin{array}{l}\stackrel{\sim}{\dot{0}} \\
\stackrel{\sim}{v}\end{array}$ & $\ddot{\infty}$ & $\begin{array}{l}0 \\
\dot{0}\end{array}$ & \begin{tabular}{l|}
0 \\
$\dot{\bullet}$ \\
\llcorner
\end{tabular} & $\cdot$. & \begin{tabular}{l}
\multirow{2}{*}{} \\
10
\end{tabular} & $\begin{array}{l}0 \\
\dot{+} \\
\dot{T}\end{array}$ & $\begin{array}{l}\stackrel{N}{\sim} \\
\stackrel{D}{\Delta} \\
\stackrel{N}{N}\end{array}$ & 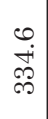 & \begin{tabular}{|l|}
$\infty$ \\
0 \\
$\dot{0}$
\end{tabular} & $\begin{array}{l}\overrightarrow{1} \\
\dot{0} \\
0\end{array}$ & $\cdot$ & $\begin{array}{l}+ \\
\infty \\
10 \\
\infty \\
\infty\end{array}$ & 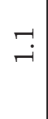 & $\begin{array}{l}\infty \\
\dot{v} \\
\stackrel{a}{*}\end{array}$ & $\ddot{0}$ & $\stackrel{0}{0}$ \\
\hline$\underset{\text { ㄱ }}{\vec{S}}$ & & $\begin{array}{l}\stackrel{N}{N} \\
-\end{array}$ & 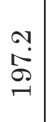 & $\vec{i}$ & $\stackrel{\overrightarrow{+}}{\stackrel{H}{\mathrm{~d}}}$ & $\vec{\infty}$ & $\ddot{0}$ & $\left|\begin{array}{l}\infty \\
\dot{1} \\
\dot{L}\end{array}\right|$ & $\cdot \cdot$ & $\begin{array}{l}\infty \\
10\end{array}$ & $\begin{array}{l}0 \\
\dot{0} \\
\llcorner\end{array}$ & \begin{tabular}{l}
$\sigma$ \\
$\infty$ \\
$\infty$ \\
$\stackrel{0}{0}$ \\
\multirow{2}{*}{}
\end{tabular} & $\begin{array}{l}\stackrel{\sim}{N} \\
\stackrel{\sim}{-} \\
\infty\end{array}$ & $\begin{array}{l}0 \\
\dot{0} \\
\end{array}$ & ㄹ. & $\begin{array}{l}10 \\
\infty\end{array}$ & 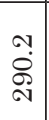 & $\stackrel{\rho}{\circ}$ & $\begin{array}{l}\mathfrak{v} \\
\mathfrak{v}\end{array}$ & $\begin{array}{l}0 \\
0\end{array}$ & $\stackrel{0}{0}$ \\
\hline 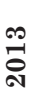 & & $\begin{array}{l}8 \\
\infty \\
0 \\
-1\end{array}$ & $\begin{array}{l}\infty \\
\stackrel{\infty}{\infty} \\
\stackrel{\sim}{-}\end{array}$ & $\stackrel{-7}{+}$ & 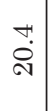 & $\stackrel{\sim}{a}$ & 0 & $\left|\begin{array}{l}\infty \\
\dot{q} \\
\dot{q}\end{array}\right|$ & $\cdot \cdot$ & $\begin{array}{l}\infty \\
10 \\
10\end{array}$ & 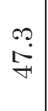 & 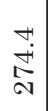 & 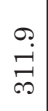 & $\begin{array}{l}\infty \\
\infty \\
\infty\end{array}$ & $\underset{⿱ 亠}{\vec{A}}$ & $\stackrel{-1}{\sim}$ & $\begin{array}{c}0 \\
\stackrel{+}{+} \\
\stackrel{N}{0}\end{array}$ & $\stackrel{\bullet}{\circ}$ & $\begin{array}{l}0 \\
\text { i }\end{array}$ & $\ddot{0}$ & $\stackrel{0}{0}$ \\
\hline 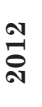 & & $\begin{array}{l}\infty \\
\stackrel{\infty}{\sim} \\
\sim\end{array}$ & 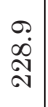 & $\begin{array}{l}10 \\
i \\
i j\end{array}$ & $\begin{array}{l}\stackrel{0}{-} \\
\stackrel{-}{-1}\end{array}$ & $\stackrel{\vec{v}}{\mathrm{j}}$ & $\begin{array}{l}0 \\
0\end{array}$ & $\left|\begin{array}{l|}\infty \\
0 \\
\dot{0} \\
\llcorner\end{array}\right|$ &.$\cdot$ & $\stackrel{\sim}{\oplus}$ & $\stackrel{\sim}{\underset{H}{+}}$ & \begin{tabular}{l}
10 \\
0 \\
0 \\
\multirow{N}{*}{} \\
\end{tabular} & $\begin{array}{l}0 \\
0 \\
0 \\
\infty \\
\infty\end{array}$ & \begin{tabular}{|l|}
$\infty$ \\
0 \\
0
\end{tabular} & $\stackrel{-1}{\stackrel{9}{N}}$ & $\underset{\sim}{\check{L}}$ & $\begin{array}{l}\infty \\
\stackrel{D}{\infty} \\
\stackrel{D}{N}\end{array}$ & $\stackrel{0}{0}$ & \begin{tabular}{|l|}
10 \\
$\stackrel{1}{*}$
\end{tabular} & $\begin{array}{l}0 \\
0\end{array}$ & $\stackrel{0}{0}$ \\
\hline$\overline{\text { อ }}$ & & $\begin{array}{l}\vec{J} \\
-7\end{array}$ & $\begin{array}{l}0 \\
\dot{0} \\
\stackrel{N}{N}\end{array}$ & $\stackrel{\circ}{\sim}$ & $\begin{array}{l}20 \\
\\
-1\end{array}$ & $\stackrel{\circ}{\mathrm{i}}$ & $\stackrel{0}{0}$ & $\left|\begin{array}{l|}\infty \\
20 \\
20\end{array}\right|$ & $\cdot \cdot$ & $\begin{array}{l}\infty \\
10 \\
10\end{array}$ & 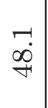 & $\begin{array}{l}10 \\
\stackrel{8}{\infty} \\
\stackrel{0}{N}\end{array}$ & 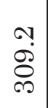 & $\stackrel{0}{\sim}$ & $\stackrel{\infty}{\stackrel{\sim}{N}}$ & $\overrightarrow{0}$ & \begin{tabular}{l}
$o$ \\
$\infty$ \\
$\infty$ \\
\multirow{\sigma}{*}{}
\end{tabular} & $\stackrel{\leftrightarrow}{0}$ & $\begin{array}{l}\tilde{N} \\
\infty \\
\infty\end{array}$ & $\stackrel{0}{0}$ & $\stackrel{0}{0}$ \\
\hline 공 & &.. & ৪ & ヘิ & 20 & ஓి & 0 & $\left|\begin{array}{l}0 \\
10\end{array}\right|$ & $\widehat{0}$ & $\stackrel{10}{\mathrm{~N}}$ & $\overrightarrow{6}$ & $\begin{array}{l}0 \\
-1\end{array}$ & $\begin{array}{l}15 \\
10 \\
00\end{array}$ & 가 & $\underset{\sim}{\infty}$ & $\cdot \cdot$ &.. & న্ల & $\frac{10}{4}$ & 0 &.. \\
\hline $\begin{array}{l}0 \\
\stackrel{0}{\circ} \\
\stackrel{-}{*}\end{array}$ & & \begin{tabular}{l}
8 \\
\hdashline \\
\hdashline
\end{tabular} & 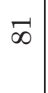 & ని & 20 & $\begin{array}{l}\infty \\
\infty \\
\infty\end{array}$ & 0 & $\underset{⿱}{\vec{F}}$ & $\overrightarrow{0}$ & $\stackrel{\infty}{\sim}$ & $\overrightarrow{0}$ & 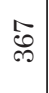 & $\mid \begin{array}{c}N \\
\infty \\
\infty \\
\infty\end{array}$ & 가 & 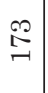 &.. & $\ddot{\mathscr{B}}$ & $F$ & 20 & 0 & 0 \\
\hline$\stackrel{10}{0}$ & $\begin{array}{l}0 \\
0 \\
.0 \\
\vdots \\
\vdots\end{array}$ & \begin{tabular}{l}
8 \\
\hdashline \\
\hdashline
\end{tabular} & 12 & $\underset{f}{\mathscr{q}}$ & î & $\approx$ & 0 & $\underset{+}{\stackrel{\leftrightarrow}{+}}$ & $\mathcal{O}$ & $\stackrel{\leftrightarrow}{\sim}$ & $\underset{\sim}{\stackrel{P}{\sim}}$ & $\begin{array}{l}0 \\
1 \\
0\end{array}$ & $\begin{array}{l}10 \\
10 \\
60\end{array}$ & $F$ & $\overrightarrow{0}$ &.. & $\underset{\sigma}{\rightleftarrows}$ & 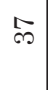 & $\begin{array}{l}0 \\
10\end{array}$ & 0 & 0 \\
\hline 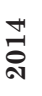 & $\begin{array}{l}\stackrel{\vec{\Xi}}{0} \\
\stackrel{0}{0} \\
\ddot{0}\end{array}$ & 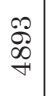 & 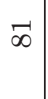 & $\stackrel{\varphi}{\not 1}$ & $\underset{+}{\infty}$ & 10 & 0 & $\underset{+}{\stackrel{F}{*}}$ & 20 & $\stackrel{\mathfrak{N}}{ }$ & $\stackrel{\sim}{\rightarrow}$ & $\frac{10}{6}$ & $\begin{array}{l}\infty \\
10 \\
\infty\end{array}$ & $\stackrel{\sim}{\rightarrow}$ & $\underset{\exists}{\sim}$ & ô & $\sqrt{-}$ & $\stackrel{\infty}{\circ}$ & $F$ & 0 & 0 \\
\hline ْి & $\begin{array}{c}\text { है } \\
\text { z } \\
\text { 乙 }\end{array}$ & 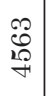 & 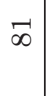 & ๙ै & ळ & م. & 0 & $\begin{array}{c}\infty \\
+\infty \\
+\end{array}$ & 20 & $\stackrel{\sim}{\circ}$ & $\begin{array}{l}\infty \\
\stackrel{\infty}{-} \\
-\rightarrow\end{array}$ & $\begin{array}{l}20 \\
10 \\
10\end{array}$ & $\stackrel{m}{\stackrel{m}{+}}$ & $\stackrel{N}{N}$ & $\begin{array}{l}\llcorner 0 \\
\stackrel{2}{\rightarrow}\end{array}$ & $\vec{\sigma}$ & $\begin{array}{l}20 \\
\stackrel{0}{0}\end{array}$ & $\underset{\sim}{\sim}$ & ஓ্ & 0 & 0 \\
\hline 로ำ & & 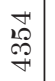 & $\begin{array}{l}10 \\
\infty\end{array}$ & $\stackrel{\Re}{\sim}$ & $\underset{\sim}{\sim} \underset{\sim}{*}$ & $\vec{\infty}$ & 0 & $\left|\begin{array}{c}0 \\
\infty \\
+ \\
+\end{array}\right|$ & 范 & 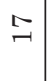 & $\begin{array}{l}\underset{\infty}{\infty} \\
\stackrel{\infty}{\sim}\end{array}$ & 算 & 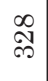 & சै & $\stackrel{\Xi}{\Xi}$ & 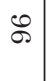 & $\overparen{\mathscr{C}}$ & 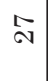 & 우 & 0 & 0 \\
\hline$\Xi$ & & 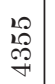 & $\begin{array}{l}10 \\
\infty\end{array}$ & $\stackrel{10}{\mathrm{~N}}$ & $\begin{array}{l}\infty \\
\infty\end{array}$ & ๙ै & 0 & $\overrightarrow{20}$ & $\stackrel{\infty}{\rightarrow+}$ & $\stackrel{\infty}{\rightarrow}$ & $\underset{+}{\stackrel{\sharp}{二}}$ & 독 & 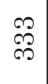 & $\begin{array}{l}0 \\
10\end{array}$ & $\stackrel{\simeq}{二}$ & $\sigma$ & श्. & 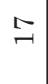 & 尺্. & 0 & 0 \\
\hline & & $\begin{array}{c}\stackrel{\infty}{N} \\
\stackrel{P}{\mid}\end{array}$ & 宫 & 空 & 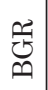 & 总 & 吕 & 뙹 & 岂 & 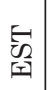 & 㕛 & 茎 & 贫 & 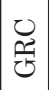 & 朰 & 疍 & $\overleftrightarrow{\Xi}$ & 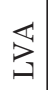 & 兒 & 兑 & 穷 \\
\hline
\end{tabular}




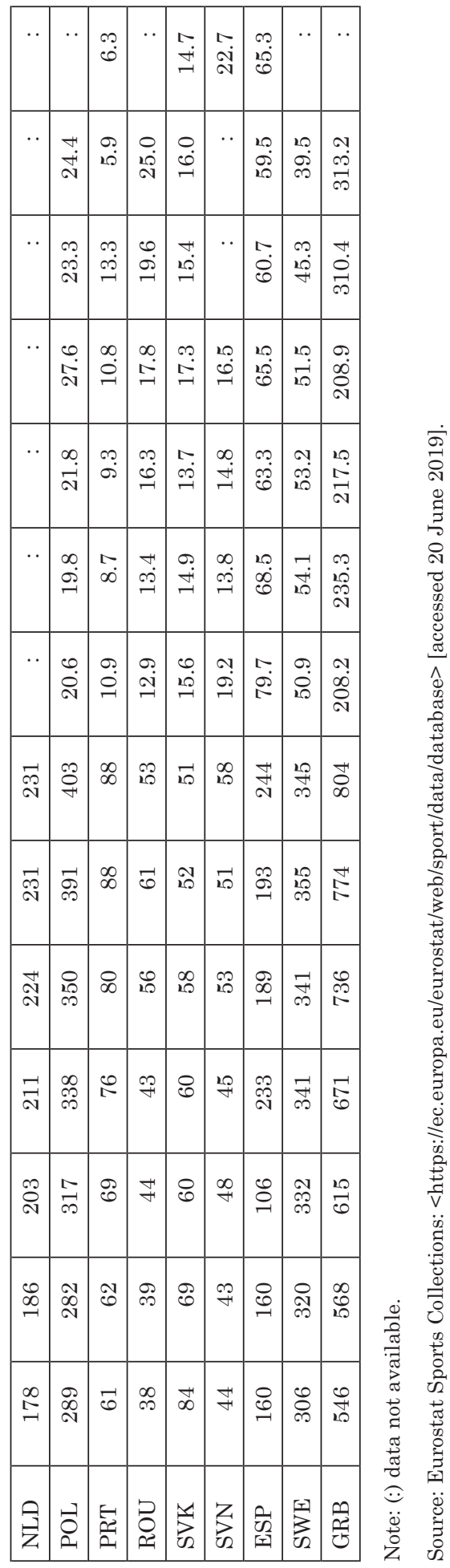




\begin{tabular}{|c|c|c|c|c|c|c|c|c|c|c|c|c|c|c|c|c|c|c|c|c|}
\hline จิ & \multirow{7}{*}{ 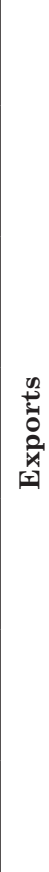 } & $\begin{array}{l}\stackrel{2}{S} \\
\text { హ. }\end{array}$ & 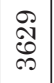 & ๙ิ & $\underset{\sim}{0}$ & กิ & $\underset{I}{\mathbb{I}}$ & 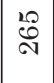 & $\mathscr{\mho}$ & 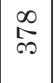 & $\begin{array}{l}\mathfrak{9} \\
\stackrel{N}{S} \\
\stackrel{D}{N}\end{array}$ & 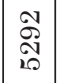 & 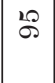 & $\stackrel{\Re}{\stackrel{+}{+}}$ & ลิ & 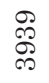 & $\stackrel{\varphi}{+1}$ & $\stackrel{m}{\stackrel{9}{=}}$ & $\infty$ & $N$ \\
\hline 㫄 & & $\underset{\infty}{*}$ & 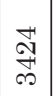 & ন & $\underset{\sim}{\stackrel{N}{O}}$ & $\mathscr{q}$ & $\begin{array}{l}\mathscr{8} \\
\mathbb{8} \\
0\end{array}$ & \begin{tabular}{|l}
$\mathscr{D}$ \\
$\stackrel{D}{N}$ \\
|
\end{tabular} & $\overline{6}$ & \begin{tabular}{|l|}
$\infty$ \\
0 \\
0 \\
$\infty$
\end{tabular} & $\left|\begin{array}{c}\infty \\
0 \\
\multirow{H}{*}{}\end{array}\right|$ & $\mid \begin{array}{l}\infty \\
10 \\
7 \\
7\end{array}$ & $\sigma$ & 告 & $\stackrel{\sim}{N}$ & $\begin{array}{l}\mathscr{2} \\
\stackrel{P}{+} \\
\infty \\
\infty\end{array}$ & $\stackrel{1}{\perp}$ & \$ & ח & 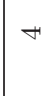 \\
\hline$\stackrel{10}{\stackrel{\text { จ }}{2}}$ & & $\begin{array}{l}\mathscr{0} \\
\infty\end{array}$ & 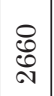 & $\stackrel{\mathscr{A}}{\mathrm{N}}$ & $\underset{\neg}{\stackrel{\leftrightarrow}{+}}$ & $\underset{\sim}{\stackrel{\sim}{\sim}}$ & \begin{tabular}{|l|}
0 \\
8 \\
10
\end{tabular} \mid & $\begin{array}{l}0 \\
\stackrel{0}{N} \\
\text { | }\end{array}$ & ชิ & $\vec{a}$ & 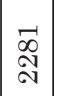 & $\left|\begin{array}{l}\mathfrak{N} \\
\stackrel{D}{D} \\
\infty \\
\infty\end{array}\right|$ & 实 & ஓ & จิ & 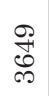 & $\mathscr{\infty}$ & $\underset{-}{\circ}$ & ח & 10 \\
\hline 㫄 & & $\begin{array}{l}20 \\
\infty \\
\infty\end{array}$ & $\begin{array}{l}\stackrel{S}{S} \\
\text { N }\end{array}$ & $\stackrel{\infty}{\stackrel{2}{\rightarrow ~}}$ & 亲 & $\mathscr{8}$ & $\underset{+}{\mathscr{F}}$ & \begin{tabular}{|l|l}
10 \\
10 \\
N
\end{tabular} & 8 & $\begin{array}{ll}10 \\
-10 \\
00\end{array}$ & 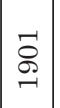 & $\left|\begin{array}{l}\overrightarrow{0} \\
0 \\
\infty \\
\infty\end{array}\right|$ & 5 & $\stackrel{\sim}{\sim}$ & ลั & \begin{tabular}{|l}
10 \\
10 \\
$\infty$ \\
$\infty$ \\
$\infty$
\end{tabular} & $\stackrel{.}{\circ}$ & $\stackrel{\infty}{\circ}$ & $\overrightarrow{\mathrm{N}}$ & 0 \\
\hline$\stackrel{\infty}{\stackrel{乛}{*}}$ & & $\underset{\infty}{\stackrel{\infty}{\llcorner}}$ & 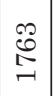 & 点 & 18 & 0 & $\begin{array}{c}\overrightarrow{0} \\
\overrightarrow{+}\end{array}$ & $\begin{array}{l}\text { \& } \\
\text { \& }\end{array}$ & $\stackrel{10}{1}$ & 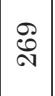 & $\begin{array}{l}2 \\
\stackrel{2}{2} \\
\stackrel{5}{-}\end{array}$ & $\left|\begin{array}{l}\vec{b} \\
0 \\
0 \\
-1\end{array}\right|$ & $\stackrel{R}{2}$ & N & $\stackrel{20}{-10}$ & 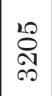 & $\stackrel{\sim}{N}$ & $\stackrel{\infty}{\circ}$ & $\overrightarrow{\mathrm{N}}$ & H \\
\hline ণ্] & & $\vec{E}$ & $\begin{array}{l}8 \\
0 \\
0 \\
-1\end{array}$ & $\stackrel{\mathfrak{N}}{\stackrel{\sim}{-}}$ & $\begin{array}{l}10 \\
10 \\
-10\end{array}$ & $\infty$ & $\left|\begin{array}{c}\mathscr{N} \\
\stackrel{F}{+} \\
\stackrel{F}{*}\end{array}\right|$ & I & 8 & 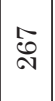 & $\left|\begin{array}{c}\vec{N} \\
\infty \\
-\infty\end{array}\right|$ & \begin{tabular}{l}
\multirow{N}{*}{} \\
Oे
\end{tabular} & $\Phi_{\infty}^{+}$ & $\underset{-\infty}{\infty}$ & 尺 & 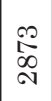 & 아 & ৪ & $\stackrel{2}{\mathrm{~N}}$ & $\stackrel{\bullet}{\sim}$ \\
\hline 귱 & & $\hat{\mathscr{Q}}$ & 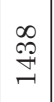 & $\stackrel{\stackrel{20}{f}}{=}$ & $\underset{-1}{8}$ & $\infty$ & 㹲 & $\overrightarrow{\text { จे }}$ & 8 & 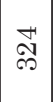 & 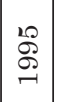 & \begin{tabular}{|c|}
0 \\
$\stackrel{D}{0}$ \\
$\stackrel{D}{N}$
\end{tabular} & $\mathscr{8}$ & 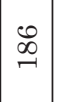 & $\vec{m}$ & $\begin{array}{l}\infty \\
0 \\
6 \\
\infty \\
\infty\end{array}$ & $\stackrel{10}{\mathrm{~N}}$ & $\mathscr{8}$ & $\stackrel{\sim}{\sim}$ & $\stackrel{2}{\rightarrow}$ \\
\hline 공 & \multirow{8}{*}{ 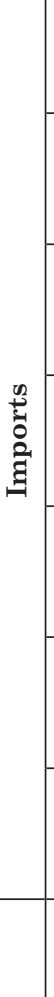 } & $\overrightarrow{\vec{N}}$ & $\begin{array}{l}\vec{b} \\
\text { N } \\
\text { N }\end{array}$ & 운 & $\begin{array}{l}\infty \\
\stackrel{\infty}{N} \\
\stackrel{N}{N}\end{array}$ & \&্নে & \begin{tabular}{|l|}
$\mathcal{1}$ \\
$\mathcal{O}$ \\
$\mathcal{1}$
\end{tabular} & 范 & 10 & 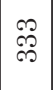 & \begin{tabular}{|l|}
10 \\
0 \\
10 \\
00 \\
0
\end{tabular} & 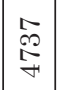 & $\stackrel{\sim}{\sim}$ & 尺े & 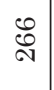 & \begin{tabular}{|l|}
20 \\
0 \\
\multirow{2}{*}{} \\
\end{tabular} & 8 & $\vec{\infty}$ & 8 & : \\
\hline 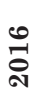 & & $\begin{array}{l}\infty \\
\vec{\sigma} \\
-\end{array}$ & 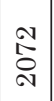 & $\ddot{8}$ & $\stackrel{\circ}{\circ}$ & \begin{tabular}{l}
$\infty$ \\
\multirow{1}{*}{}
\end{tabular} & $\begin{array}{l}0 \\
i 0\end{array}$ & $\overrightarrow{0}$ & $\stackrel{\infty}{+}$ & 苨 & $\left|\begin{array}{l}10 \\
10 \\
\infty \\
\infty \\
\infty\end{array}\right|$ & $\mid \begin{array}{l}\vec{b} \\
\stackrel{f}{\forall}\end{array}$ & $\mid \begin{array}{l}\infty \\
\infty \\
\stackrel{\infty}{N}\end{array}$ & 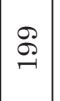 & $\overrightarrow{\mathscr{N}}$ & 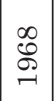 & \begin{tabular}{|l|}
10 \\
10
\end{tabular} & $F$ & is & \begin{tabular}{l}
$\infty$ \\
\hdashline \\
$\bullet$
\end{tabular} \\
\hline 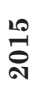 & & 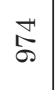 & 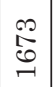 & ำ & $\begin{array}{l}0 \\
\stackrel{N}{N}\end{array}$ & H & $\stackrel{8}{\circ}$ & $\begin{array}{l}\stackrel{9}{2} \\
\stackrel{4}{+}\end{array}$ & $\stackrel{\infty}{+}$ & 离 & $\begin{array}{l}\stackrel{\mathscr{N}}{2} \\
\stackrel{\sim}{N}\end{array}$ & $\mid \begin{array}{l}\stackrel{\mathscr{D}}{\mathrm{D}} \\
\stackrel{\text { f }}{\mid}\end{array}$ & 命 & 占 & $\begin{array}{l}10 \\
\stackrel{2}{N}\end{array}$ & 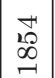 & 201 & $\overrightarrow{0}$ & ชै & 돗 \\
\hline ন্ & & $\vec{\sigma}$ & $\begin{array}{l}\text { 点 } \\
\underset{-}{*}\end{array}$ & $\mid \begin{array}{l}\infty \\
10\end{array}$ & $\stackrel{\circ}{\stackrel{一}{\prime}}$ & 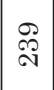 & Iै & $\begin{array}{l}0 \\
\frac{6}{7} \\
\end{array}$ & 아 & $\begin{array}{c}20 \\
\stackrel{2}{N} \\
\text { N }\end{array}$ & $\begin{array}{c}\infty \\
+1 \\
\stackrel{1}{N}\end{array}$ & $\begin{array}{l}0 \\
\vec{\sigma} \\
\infty\end{array}$ & ㄱ. & $\begin{array}{l}\infty \\
\stackrel{\infty}{\rightarrow} \\
\stackrel{\rho}{-1}\end{array}$ & $\underset{\mathscr{C}}{\mathscr{\bullet}}$ & 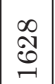 & 足 & $\overrightarrow{6}$ & i⿱ & $\begin{array}{l}\infty \\
\stackrel{\sim}{+}\end{array}$ \\
\hline ঙ্ণ & & $\begin{array}{l}0 \\
\stackrel{D}{\infty} \\
\infty\end{array}$ & 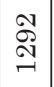 & $\stackrel{\infty}{+}$ & 令 & 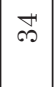 & $\begin{array}{l}\overrightarrow{0} \\
\text {. }\end{array}$ & 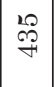 & $\not{F}$ & $\begin{array}{l}\infty \\
\ddot{\infty}\end{array}$ & 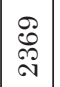 & $\begin{array}{l}0 \\
\stackrel{1}{1} \\
\end{array}$ & $\underset{10}{10}$ & $\stackrel{20}{\circ}$ & 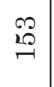 & $\begin{array}{l}0 \\
\text { 암 } \\
\text { on } \\
-1\end{array}$ & $\stackrel{\infty}{+}$ & 各 & ชै & $\begin{array}{l}0 \\
2 \\
\infty\end{array}$ \\
\hline$\stackrel{\text { จิ }}{\stackrel{\text { จे }}{2}}$ & & $\stackrel{\infty}{\stackrel{\infty}{\llcorner}}$ & $\begin{array}{l}\vec{F} \\
\vec{m}\end{array}$ & 우 & 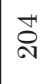 & $\stackrel{\mathscr{F}}{\forall}$ & $\vec{S}$ & \begin{tabular}{|c}
$\hat{\kappa}$ \\
$\stackrel{2}{q}$
\end{tabular} & $\stackrel{\mathfrak{F}}{\forall}$ & 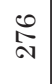 & 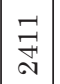 & $\mid \begin{array}{l}20 \\
\stackrel{\mathbb{N}}{\mathrm{N}}\end{array}$ & $\begin{array}{l}\mathscr{8} \\
0 \\
0\end{array}$ & $\infty$ & $\begin{array}{l}0 \\
\stackrel{10}{\Omega} \\
\sim\end{array}$ & \begin{tabular}{|c|}
0 \\
0 \\
10 \\
$\sim$
\end{tabular} & 8 & 아 & 20 & $\underset{\infty}{\stackrel{N}{N}}$ \\
\hline $\overrightarrow{\text { จे }}$ & & 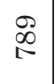 & 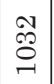 & $\stackrel{\infty}{+}$ & 등 & 아 & $\underset{:}{\stackrel{N}{*}}$ & 草 & 全 & 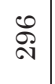 & 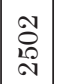 & $\mid \begin{array}{l}\infty \\
\stackrel{8}{2} \\
\stackrel{2}{N}\end{array}$ & $\mid \begin{array}{l}+ \\
\infty \\
\sim\end{array}$ & హ゙ & 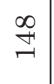 & 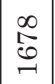 & $\underset{+}{*}$ & 苟 & \begin{tabular}{|l}
$\infty$ \\
10 \\
10
\end{tabular} & $\vec{S}$ \\
\hline & & 宫 & 国 & 总 & 总 & $\underset{\partial}{2}$ & 종 & 嵏 & $\begin{array}{l}\vec{E} \\
\tilde{n} \\
\vec{r}\end{array}$ & 尝 & 死 & 只 & $\begin{array}{l}\text { O } \\
\text { 秀 }\end{array}$ & 兄 & 茎 & $\overleftrightarrow{\Xi}$ & 岁 & 虽 & 崌 & 㿟 \\
\hline
\end{tabular}




\begin{tabular}{|c|c|c|c|c|c|c|c|c|}
\hline $\begin{array}{l}0 \\
\mathscr{\delta} \\
\infty\end{array}$ & $\begin{array}{l}\stackrel{9}{I} \\
ت \\
-1\end{array}$ & $\underset{+}{\stackrel{丶}{2}}$ & $\underset{\infty}{\stackrel{N}{N}}$ & $\stackrel{10}{\stackrel{2}{-}}$ & $\stackrel{10}{-10}$ & 苑 & $\begin{array}{l}\stackrel{0}{0} \\
\text { D. }\end{array}$ & $\begin{array}{l}0 \\
\sigma \\
\sigma\end{array}$ \\
\hline $\begin{array}{l}8 \\
\infty \\
\infty\end{array}$ & $\stackrel{\infty}{\infty}$ & 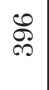 & 告 & $\vec{\sigma}$ & $\stackrel{\text { ลิ }}{\sim}$ & $\underset{\infty}{\stackrel{-}{\infty}}$ & F & $\begin{array}{l}\infty \\
\stackrel{\infty}{5} \\
\stackrel{\circ}{\varsigma}\end{array}$ \\
\hline $\begin{array}{l}\not{D} \\
\text { ON } \\
\infty\end{array}$ & 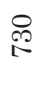 & 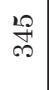 & $\begin{array}{l}\text { 点 } \\
\text { ch }\end{array}$ & $\begin{array}{l}\infty \\
\infty \\
\sim\end{array}$ & $\stackrel{0}{=}$ & $\begin{array}{l}0 \\
\infty \\
\infty\end{array}$ & 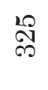 & $\stackrel{10}{\stackrel{10}{\circ}}$ \\
\hline $\begin{array}{l}\infty \\
\stackrel{1}{1} \\
\sim \\
-\end{array}$ & $\begin{array}{l}0 \\
i 0\end{array}$ & $\underset{\infty}{\vec{\Delta}}$ & $\vec{ర}$ & 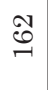 & $\underset{ت}{\stackrel{\Xi}{二}}$ & $\begin{array}{l}0 \\
0\end{array}$ & $\begin{array}{l}\stackrel{D}{\infty} \\
\stackrel{N}{N}\end{array}$ & 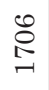 \\
\hline 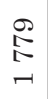 & $\begin{array}{l}\infty \\
\stackrel{0}{\mathscr{H}}\end{array}$ & $\underset{\sim}{\stackrel{H}{N}}$ & $\stackrel{\infty}{\stackrel{\infty}{\infty}}$ & $\underset{+}{\stackrel{H}{-}}$ & $\underset{\exists}{\stackrel{J}{二}}$ & 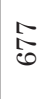 & $\begin{array}{l}10 \\
\infty \\
\text { Do }\end{array}$ & $\begin{array}{l}\infty \\
\infty \\
10 \\
\end{array}$ \\
\hline $\begin{array}{l}\mathscr{8} \\
\infty \\
-1\end{array}$ & $\begin{array}{l}\infty \\
\stackrel{N}{*}\end{array}$ & $\stackrel{\rho}{\circ}$ & $\underset{\infty}{\stackrel{\sim}{~}}$ & $\stackrel{\text { مै }}{\rightarrow}$ & $\stackrel{10}{\stackrel{10}{-1}}$ & 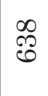 & $\stackrel{\infty}{\stackrel{\infty}{\sim}}$ & $\underset{\sim}{\stackrel{2}{2}}$ \\
\hline 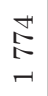 & $\vec{ఠ}$ & $\overrightarrow{\mathrm{S}}$ & $\begin{array}{l}\infty \\
\infty \\
\infty \\
\infty\end{array}$ & $\begin{array}{l}\stackrel{10}{\mathrm{~N}} \\
\stackrel{\sim}{\sim}\end{array}$ & $\stackrel{\leftrightarrow}{\stackrel{\leftrightarrow}{\rightarrow}}$ & 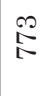 & $\begin{array}{l}10 \\
\stackrel{10}{N} \\
\text { Non }\end{array}$ & $\begin{array}{l}\infty \\
\stackrel{1}{10} \\
\end{array}$ \\
\hline 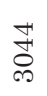 & $\stackrel{\mathscr{P}}{\infty}$ & $\frac{\infty}{\infty}$ & $\mid \begin{array}{l}\infty \\
\infty \\
-1\end{array}$ & $\stackrel{\vec{S}}{\stackrel{S}{\circ}}$ & $\overrightarrow{0}$ & 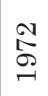 & $\frac{10}{\sigma}$ & $\begin{array}{l}10 \\
10 \\
10 \\
0\end{array}$ \\
\hline $\begin{array}{l}\frac{10}{\sigma} \\
\frac{\sigma}{N}\end{array}$ & $\stackrel{\mathscr{N}}{N}$ & $\stackrel{\infty}{\stackrel{\leftrightarrow}{S}}$ & 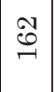 & $\stackrel{\curvearrowright}{\stackrel{\curvearrowright}{\sim}}$ & $\begin{array}{l}\stackrel{0}{\sim} \\
\stackrel{\sim}{\sim}\end{array}$ & $\begin{array}{l}\stackrel{N}{1} \\
\stackrel{1}{5} \\
-1\end{array}$ & $\stackrel{\sim}{\infty}$ & $\underset{\infty}{\stackrel{\odot}{\rightleftarrows}}$ \\
\hline $\begin{array}{l}10 \\
\infty \\
上 \\
-1\end{array}$ & $\begin{array}{l}\infty \\
\infty \\
10\end{array}$ & $\stackrel{20}{\stackrel{\sim}{H}}$ & 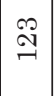 & $\stackrel{\substack{\llcorner \\
\sim}}{ }$ & $\stackrel{g}{\leftrightarrows}$ & $\begin{array}{l}\text {-0 } \\
\stackrel{0}{10} \\
-1\end{array}$ & શิ & $\begin{array}{l}10 \\
6 \\
\infty\end{array}$ \\
\hline$\stackrel{\leftrightarrow}{\stackrel{8}{\circ}}$ & $\underset{ }{\stackrel{O}{4}}$ & $\underset{N}{\stackrel{N}{N}}$ & $\exists$ & $\underset{+}{\vec{I}}$ & $\stackrel{\circ}{\stackrel{\circ}{ }}$ & $\begin{array}{l}\stackrel{N}{0} \\
\stackrel{+}{-} \\
-\end{array}$ & $\begin{array}{l}\infty \\
\mathscr{0}\end{array}$ & 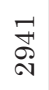 \\
\hline$\stackrel{\stackrel{\mathscr{N}}{\sim}}{\rightarrow}$ & $\begin{array}{l}\infty \\
10 \\
\infty\end{array}$ & : 20 & $\begin{array}{l}\infty \\
\curvearrowright\end{array}$ & $\stackrel{20}{\underset{f}{二}}$ & $\stackrel{8}{\circ}$ & 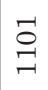 & $\begin{array}{l}10 \\
10 \\
6\end{array}$ & 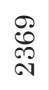 \\
\hline 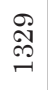 & $\begin{array}{l}0 \\
\stackrel{0}{\infty} \\
\infty\end{array}$ & $\begin{array}{l}\infty \\
\stackrel{+}{\sim} \\
\text { 出 }\end{array}$ & 命 & $\overrightarrow{\underline{m}}$ & $\underset{\sim}{\stackrel{1}{~}}$ & 용 & ญै & $\underset{\sim}{\stackrel{\infty}{H}}$ \\
\hline 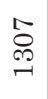 & $\vec{\infty}$ & $\underset{\sim}{\vec{H}}$ & ชু & $\underset{\sim}{\stackrel{\overbrace{}}{\sim}}$ & $\exists$ & 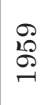 & $\frac{1}{6}$ & $\begin{array}{l}\infty \\
10 \\
\stackrel{1}{N}\end{array}$ \\
\hline 㝵 & ڤ్ & 左 & 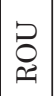 & ${ }_{0}^{4}$ & 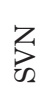 & $\begin{array}{l}0 \\
0 / 2 \\
|r|\end{array}$ & $\sum_{0}^{5}$ & 窇 \\
\hline
\end{tabular}


partners located all around the world. Belgium and the Netherlands (for NLD data on value added was not available) complete the front ranks of international trade in our sample. Sport manufacturers sell their products abroad and buy raw materials and semi-final products form foreign markets (where a cheaper work force is located). This explains why usually a high volume of imports accompanies a high volume of exports. In some economies like Poland, Hungary, Romania, and Spain, exports rocketed during the research period covered.

The mean consumption expenditure of households on sporting goods and services (Table 5) are distinguished according to the Classification of Individual Consumption According to Purpose (COICOP). It is the most commonly applied classification used to derive a consumption basket of households applicable for calculating general price level (inflation or deflation) changes over time. The sports goods and services section has subcategories: Major durables for outdoor (1) and indoor recreation (2), Maintenance and repair of other major durables for recreation and culture (3), Equipment for sport, camping and open-air recreation (4), Recreational and sporting services - attendance and participation (6). We present the total mean expenditure of private households and juxtapose it with spending on sports goods and services. The Eurostat sports' data collection provides statistics only for 2010 and 2015.

Table 5

Consumption expenditure of households on sporting goods and services

\begin{tabular}{|l|c|c|l|c|c|}
\hline MSs & $\begin{array}{c}\text { Total } \\
\text { consumption }\end{array}$ & $\begin{array}{c}\text { Sports goods } \\
\text { and services } \\
\text { consumption }\end{array}$ & MSs & $\begin{array}{c}\text { Total } \\
\text { consumption }\end{array}$ & $\begin{array}{c}\text { Sports goods } \\
\text { and services } \\
\text { consumption }\end{array}$ \\
\hline AUT & 33219 & 493.3 & BEL & 31264 & 358.3 \\
\hline BGR & 9334 & 9.2 & HRV & 17264 & 66.0 \\
\hline CYP & 44641 & 374.3 & CZE & 13161 & 162.9 \\
\hline DNK & 28560 & 286.7 & EST & 10421 & 135.2 \\
\hline FIN & 26998 & 609.4 & FRA & 27627 & 308.7 \\
\hline DEU & 28367 & 350.0 & GRC & 29974 & 157.2 \\
\hline HUN & 14017 & 61.0 & IRL & 33262 & 469.0 \\
\hline ITA & 28393 & 205.3 & LVA & 11381 & 69.8 \\
\hline LTU & 14730 & 51.6 & LUX & 45171 & 427.6 \\
\hline MLT & 26590 & 210.1 & NLD & 30288 & $:$ \\
\hline POL & 15263 & 74.2 & PRT & 23357 & 104.0 \\
\hline ROU & 9623 & 8.5 & SVK & 15041 & 261.6 \\
\hline SVN & 25514 & 339.7 & ESP & 30884 & 539.5 \\
\hline SWE & 26366 & 637.2 & GRB & 23692 & \\
\hline
\end{tabular}

Note: (:) data not available.

Source: Eurostat Sports Collections: <https://ec.europa.eu/eurostat/web/sport/data/database> [accessed 20 June 2019]. 


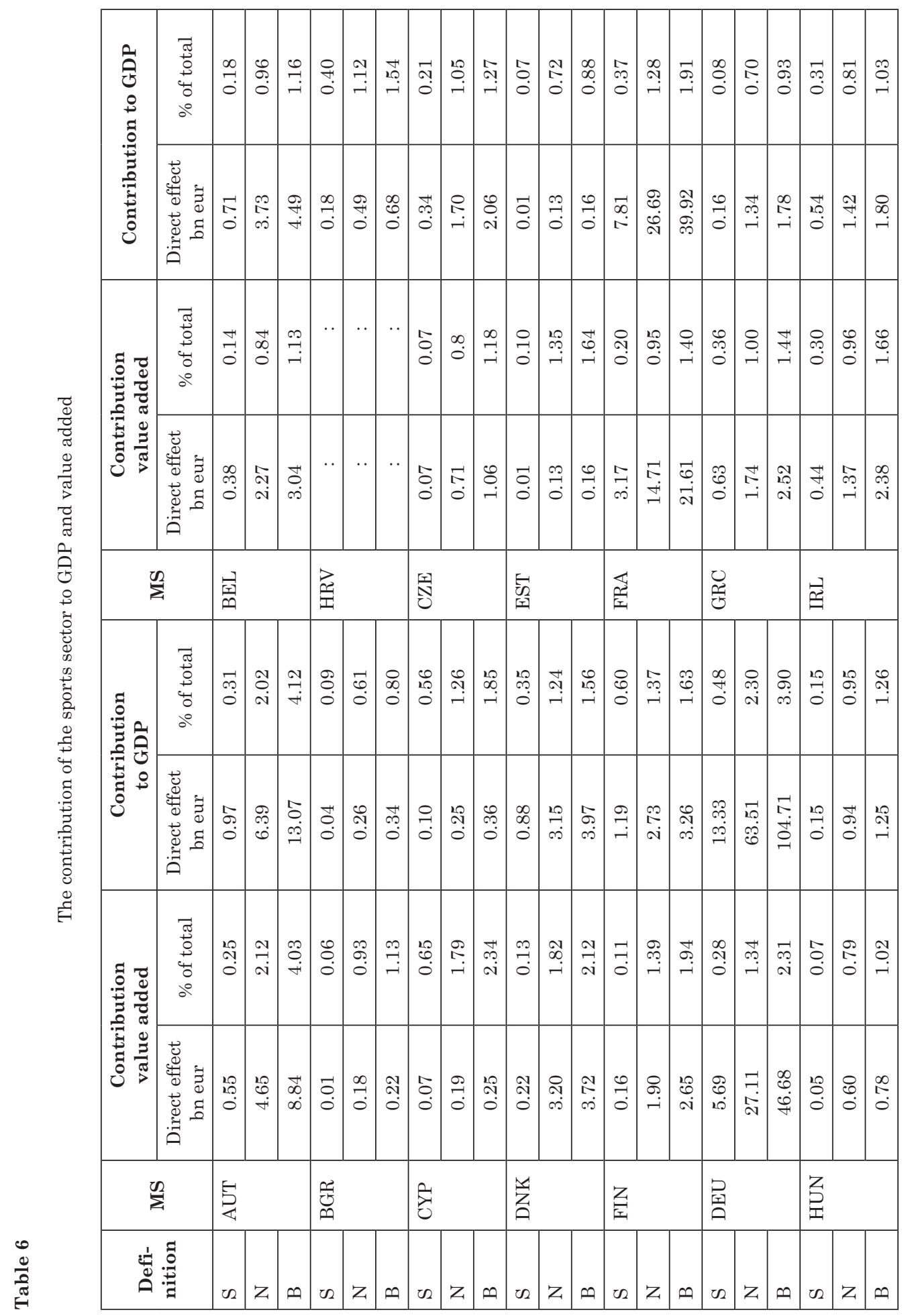




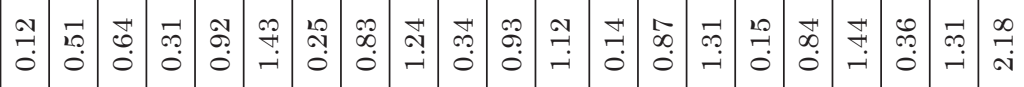

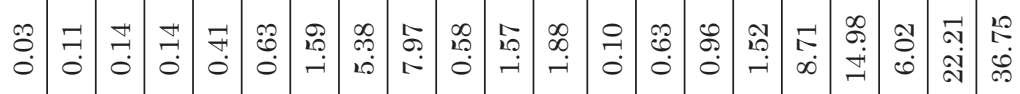

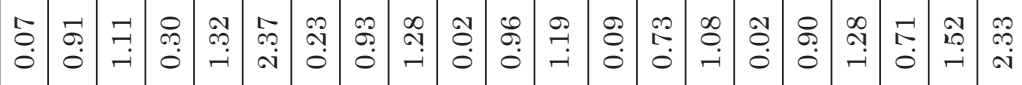

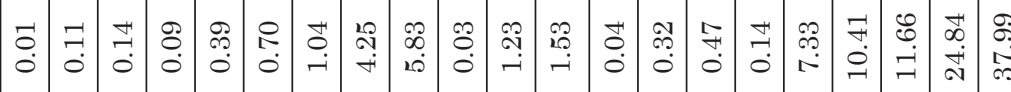

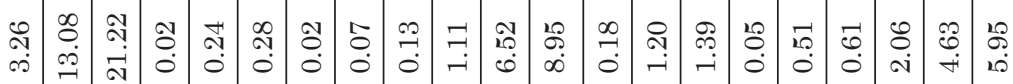

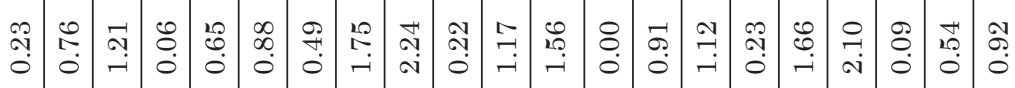

$\dot{\sigma}$
$\stackrel{\circ}{\Xi}$
$\stackrel{\Xi}{\Xi}$
$\stackrel{0}{0}$

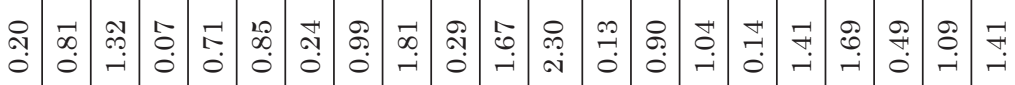

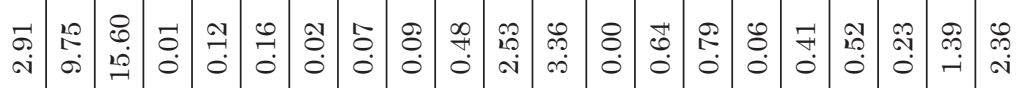

\begin{tabular}{|c|c|c|c|c|c|c|c|c|c|c|c|c|c|c|c|c|c|c|c|c|}
\hline \multicolumn{3}{|l|}{ 崩 } & \multicolumn{3}{|c|}{ • } & \multicolumn{3}{|c|}{ 实 } & \multicolumn{3}{|l|}{$\stackrel{7}{\circ}$} & \multicolumn{3}{|l|}{ 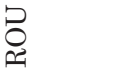 } & \multicolumn{3}{|l|}{$\frac{z}{z}$} & \multicolumn{3}{|l|}{$\frac{1}{\pi}$} \\
\hline US & Z & $\varphi$ & $\pi \Omega$ & Z & 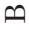 & $\boldsymbol{U}$ & Z & $\varphi$ & $\pi$ & Z & $\varphi$ & $\pi \Omega$ & 乙 & $\varphi$ & US & Z & $\varphi$ & $\pi \Omega$ & Z & 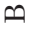 \\
\hline
\end{tabular}

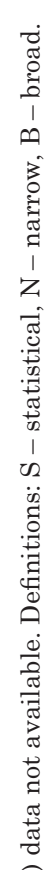

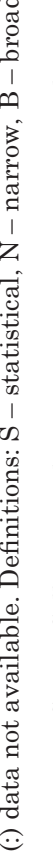

ఫัँ

$\stackrel{0}{\dot{0}}$ 
The mean households consumption of sport goods and services per year was relatively small compared to total income: it varies between $0.1 \%$ and 2.5\%. It increased between 2010 and 2015 in the majority of cases. That is not surprising as in the majority of cases total consumption was also higher. However, there are some exceptions: Cyprus, Greece and Spain experienced prolonged consequences due to the Great Recession - deep and persistent economic downturn - which is also reflected in a decline in total expenditures. Reducing spending on sport goods and services is a natural option for difficult times. It was also an option for some better performing economies from our sample. The most significant increase in spending on sport goods and services was registered in transforming economies: for instance it was tripled for Bulgaria and doubled for Croatia, Estonia and Latvia. Room for their growth exists in lower income MSs.

Finally, we present the results of sport's contribution to the gross domestic products of national economies and its gross value added. Table 6 is based on two Reports whose methodology was briefly described in the previous section (SPeA, 2012 and SPeA, 2018). The estimations are based on the Vilnius definition of sports: statistical, narrow and broad. Reports are based on 2012 data - more recent estimations are not available due to the lack of a more upto-date SSAs release.

As each of the definitions expands the meaning of sport, it is obvious that contribution of the sports to gross value added or GDP amounts to below 1\% for both measures of aggregate economic activity when the statistical definition is covered. It increases for the narrow and broad definitions of sports. Cross-country differences of sport's economic importance emerge. Three EU MSs with the most important contribution of sports sector in relative terms were: Austria, the UK, and Germany. The ranging-closing MSs are: Lithuania, Latvia and Bulgaria. This ranking mimics to some extent the importance of the sports sector expressed by Eurostat statistics. The share of sports-related value added for the European Union is $1.13 \%$ for the narrow definition, and $1.76 \%$ for the broad definition of sport. For the statistical definition it is only about $0.28 \% .^{23}$ This means that the official statistical approach to sports which is reflected in its statistical definition covers only about one sixth of its real value. The contribution of sports to GDP on the European level was about $2.12 \%$ of total GDP. ${ }^{24}$ For both statistics we can find MSs being remarkably below and above the EU average.

23 SportsEconAustria (SpEA), Study on the Contribution of Sport to Economic Growth and Employment in the EU, 2012, < https://publications.europa.eu/en/publication-detail/-/publication /865ef44c-5ca1-11e8-ab41-01aa75ed71a1/language-en/format-PDF/source-71256399> [accessed 15 June 2019]: 77.

24 SportsEconAustria (SpEA), Study on the Economic Impact of Sport through Sport Satellite Accounts, 2018, <https://ec.europa.eu/assets/eac/sport/library/studies/study-contribution-spors-economic-growth-final-rpt.pdf> [accessed 15 June 2019]: 15. 


\section{CONCLUSIONS}

In this examination we sought to compare the macroeconomic importance of the sports sector in European Union Member States. This issue is worth examination for several reasons: (i) it is difficult, as various ways of defining sport exist; (ii) as a consequence, official statistics do not cover the entire sports sector; (iii) sport contributes to the national economy in various ways and has gained more importance over time - its estimation is more important than few decades ago; (iv) this topic has not been examined thoroughly. Cross-country comparison is missing. Thus our paper built on the existing literature, as we provide the characteristics of available sources of sports sector measurement and a multisource international comparison of the importance of the sports sector.

The results for the EU MSs indicate the existence of some economies that are more sports-based. These are mostly developed economies. However, even for them, the sports sector is far away from double-digit contribution to GDP or value added. We also observe that the importance of sports increases over time, although some exceptions exist.

We need to mention some caveats of our study. We discuss the data provided by Eurostat, which are based on a statistical definition of sport. The Eurostat sports collections have been provided for quite a short period of time. Still, this database is more consistent and regular than the Sport Satellite Accounts and SSA-based examinations. The overall picture of the sports sector could not be drawn due to data accessibility. Even SSAs based reports that provide more consistent estimations of the sports sector are not flawless: they need a set of assumptions to be made for the economic relevance of sports to be discovered.

Finally, we see some potential extensions of our examination. In depth individual country level analysis would be the first one. Secondly, we could incorporate the results of the newest SSAs examination which are just about to be published. As sport represents a large and fast-growing sector of national economies, the tools designed to measure its economic importance need to be elaborated.

Mariola Mamcarczyk

Cracow University of Economics

mamcarcm@uek.krakow.pl

https://orcid.org/0000-0003-0316-533X

Magdalena Szyszko

WSB University in Poznan

magdalena.szyszko@wsb.poznan.pl

https://orcid.org/0000-0002-5322-3334

Andreff, W. (2008). Globalization of the sport economy. Rivista Di Diritto ed Economica dello Sport 4(3): 13-32.

Allmers, S., Maennig, W. (2009). Economic impacts of the FIFA Soccer World Cups in France 1998, Germany 2006, and outlook for South Africa 2010. Eastern Economic Journal 35(4): $500-519$. 
Cyganiuk, J. (2011). Aktywność ruchowa jako skuteczny środek profilaktyki zdrowotnej i promocji zdrowia młodzieży gimnazjalnej, [w:] M. Jurgielewicz-Urniaż (ed.), Sport i wychowanie zdrowotne, Olsztyńska Szkoła Wyższa im. Józefa Rusieckiego. Olsztyn: 85.

Grabowski, A. (2014). Znaczenie ekonomiczne sportu w Unii Europejskiej. Studia Ekonomiczne 176(14): 189-200.

Grabowski, A. (2017). Analiza Rachunku Satelitarnego Sportu w krajach Unii Europejskiej. Studia Ekonomiczne 316: 94-102.

Gibson, H., McIntyre, S., MacKay, S., Riddington G. (2005). The economic impact of sports, sporting events, and sports tourism in the U.K. The DREAM ${ }^{\mathrm{TM}}$ Model. European Sport Management Quarterly 5(3): 321-332.

Gratton, Ch. (1998). The economic importance of modern sport. Culture, Sport, Society 1(1): 101117.

Jakobsen, J., Solberg, H.A., Halvorsen, T., Jakobsen, T.G. (2013). Fool's gold: major sport events and foreign direct investment. International Journal of Sport Policy and Politics 5(3): 363380. doi:10.1080/19406940.2012.71709

Kozłowska, E., Kowalczyk, A., Rząca, M., Kocka, K. (2015). Uczestnictwo w lekcjach wychowania fizycznego a rozwój kultury fizycznej po zakończeniu etapu edukacyjnego (Participation in physical education classes and development of the physical culture after educational stage). Journal of Education, Health and Sport 5(4): 355-365.

Matheson, V.A. (2009). Economic multipliers and mega-event analysis. International Journal of Sport Finance 4(1): 63-70.

Ministerstwo Sportu i Turystyki (2017). Rachunek Satelitarny Sportu dla Polski.

Porter, P.K., Fletcher, D. (2008). The economic impact of the Olympic Games: ex ante predictions and ex post reality. Journal of Sport Management 22(4): 470-486.

Rose, A.K., Spiegel, M.M. (2011). The Olympic effect. Economic Journal 121(553): 652-677.

Siwiński, W., Tauber, R.D., Mucha-Szajek, E. (2009). Wpływ światowego kryzysu finansowego na rozwój sportu, turystyki i rekreacji. Poznań.

\title{
THE MACROECONOMIC ASPECTS OF SPORT IN EUROPE: A CROSS-COUNTRY COMPARISON
}

\author{
Sum mary
}

In this paper we aim to investigate the importance of the sports sector of the economy for macrolevel performance in the European Union Member States. The problem that we address is worthy of research focus as sport is a dynamically expanding and important sector of the economy. The increasing importance of sport in national economies cannot be fully analysed due to the lack of sufficient statistical data. In this paper, we provide a comparison of the Eurostat sports collections results and Sport Satellite Account-based examination of the contribution of sport to the GDP and value added. The analysis of statistical data is preceded by a description of the sports sector measurement. We establish that the contribution of sport is more remarkable for developed economies, however, it has also been increasing over time in other EU Member States. This can be observed for employment, changes in enterprises' statistics, and international trade. Household spending on sports goods and services also increases even if the Great Recession led to a downturn in sport consumption in some countries. The frequency and coherence of sports data collections related to its economic significance is not satisfactory. The issue needs to be given a higher priority by public authorities.

Keywords: sport economy; economic effects of sports; statistical data; JEL codes: Z29, E01, L83 
